\title{
NUMERICAL SCHEME FOR THE ONE-PHASE 1D STEFAN PROBLEM USING CURVILINEAR COORDINATES
}

\author{
Marek Blasik \\ Institute of Mathematics, Czestochowa University of Technology \\ Czestochowa, Poland \\ marek.blasik@im.pcz.pl
}

\begin{abstract}
In this paper we present a new approach to solving a one-dimensional, one-phase Stefan problem. The proposed method is based on choosing (a) suitable curvilinear space coordinate/s for the heat-flow equation and the finite difference method. In the final part of this paper, examples of numerical calculations are shown.
\end{abstract}

\section{Introduction}

Moving boundary problems in which the boundary of the domain is not known occur in subjects such as heat flow, hydrology, solute transport or molecular diffusion. Moving boundaries are associated with time dependent problems and the position of the boundary has to be determined as a function of time. Moving boundary problems are often called Stefan problems, with reference to the work of J. Stefan, who investigated the melting of the polar ice cap [1-3].

In the literature there are many well-known methods for solving the Stefan problem. One of them, proposed by Schniewind, is based on the concept that moving boundary moves "from node to node" $[4,5]$. A similar approach, where the moving boundary always moves from one grid point to another, was applied by Douglas and Gallie in [6]. These are the methods with variable time step. Murray and Landis suggested a different approach [7]. Their method uses a variable space grid with constant number of space intervals between fixed and moving boundary. Another front-tracking method was proposed by Crank [8]. In his method, the moving boundary will usually be located between two neighbouring grid points. The solution is determined by using a modified finite-difference method which incorporates unequal space intervals near moving boundary. One of the most popular methods of solving moving boundary problems is fixing the moving boundary at the same grid point or line for all time by a suitable coordinate transformation $[2,8]$.

Our aim is to construct a numerical scheme which allows the use of a constant both the time and the space steps. In this approach we expect the boundary to move along a straight line.

The paper is organized as follows. In the first section we formulate one-dimensional, one-phase Stefan problem in curvilinear coordinates. Numerical scheme 
is given in Section 2. Then, two examples of the simulations are presented in Section 3.

\section{Mathematical formulation of the problem}

Consider a simple version of the Stefan problem describing melting of a semiinfinite sheet:

$$
c \rho \frac{\partial u(x, t)}{\partial t}=K \frac{\partial^{2} u(x, t)}{\partial x^{2}}, \quad 0<x<s(t), \quad t>0
$$

with initial-boundary conditions

$$
\begin{gathered}
u(0, t)=u_{0}, \quad u(s(t), t)=u_{B}, \quad t>0, \\
u(0,0)=u_{B},
\end{gathered}
$$

and with Stefan condition

$$
L \rho \frac{d s(t)}{d t}=-\left.K \frac{\partial u(x, t)}{\partial x}\right|_{x=s(t)}, \quad s(0)=0,
$$

where $K$ is conductivity, $u$ is temperature, $\rho$ is density, $c$ is specific heat and $L$ is the latent heat required to melt the given material. Assuming constant thermal values and using a simple scaling:

$$
X=\frac{x}{l}, \quad \tau=t \frac{K}{c \rho l^{2}}, \quad \tilde{U}=\frac{u}{u_{0}}, \quad \tilde{S}=\frac{s}{l}
$$

we can formulate the above Stefan problem in non-dimensional variables for finite sheet $0 \leq x \leq l$, where $l$ is some standard length and $\Lambda=c u_{0} / L$ is dimensionless Stefan number:

$$
\begin{gathered}
\frac{\partial \tilde{U}(X, \tau)}{\partial \tau}=\frac{\partial^{2} \tilde{U}(X, \tau)}{\partial X^{2}}, \quad 0<X<\tilde{S}(\tau) \\
\tilde{U}(0, \tau)=1, \quad \tilde{U}(\tilde{S}(\tau), \tau)=U_{B}, \quad \tau>0, \\
\tilde{U}(0,0)=U_{B} \\
\frac{d \tilde{S}(\tau)}{d \tau}=-\left.\Lambda \frac{\partial \tilde{U}(X, \tau)}{\partial X}\right|_{X=\tilde{S}(\tau)}, \quad \tilde{S}(0)=0 .
\end{gathered}
$$

The analytical solution obtained by Neumann [2, 3] for the one-dimensional Stefan problem shows that the liquid/solid interface grows as the square root of 
time, namely $\tilde{S}(\tau) \sim \sqrt{\tau}$. By introducing space coordinates $\xi=X^{2}$ to the system (5)-(7) we obtain relation $S(\tau) \sim \tau$. Using standard operations we transform system (5)-(7) for function $\tilde{U}(X, \tau)$ into the following system for $U(\xi, \tau)$

$$
\begin{array}{cl}
\frac{\partial U(\xi, \tau)}{\partial \tau}=4 \xi \frac{\partial^{2}}{\partial \xi^{2}} U(\xi, \tau)+2 \frac{\partial U(\xi, \tau)}{\partial \xi}, & 0<\xi<S(\tau) \\
U(0, \tau)=1, \quad U(S(\tau), \tau)=U_{B}, & \tau>0 \\
\tilde{U}(0,0)=U_{B} & \\
p=\frac{d S(\tau)}{d \tau}=-\left.4 \xi \Lambda \frac{\partial U(\xi, \tau)}{\partial \xi}\right|_{\xi=S(\tau)=p \tau}, & S(0)=0
\end{array}
$$

where the boundary now moves along a straight line $S(\tau)=p \tau$.

\section{Numerical scheme}

Now we present a numerical scheme for the system (8)-(10). By $U_{i, j}$ we denote the values of temperature in grid points $(i \Delta \xi, j \Delta \tau)$, where $i:=0,1, \ldots, j$; $j:=0,1, \ldots, n, \Delta \xi=1 / n$ with an integer $n$. To calculate the time step $\Delta \tau$ we use formula:

$$
\Delta \tau=(n p)^{-1}
$$

where parameter $p$ is determined by solving the following transcendental equation:

$$
\frac{\Lambda}{\sqrt{\pi}}\left(1-\frac{u_{B}}{u_{0}}\right)=\frac{\sqrt{p}}{2} \exp \left(\frac{p}{4}\right) \operatorname{erf}\left(\frac{\sqrt{p}}{2}\right) .
$$

To approximate derivatives from formula (8) we use the difference quotients and transform the equation (8) to the following discrete form

$$
\frac{U_{i, j}-U_{i, j-1}}{\Delta \tau}=4 i \Delta \xi \frac{U_{i-1, j}-2 U_{i, j}+U_{i+1, j}}{\Delta \xi^{2}}+2 \frac{U_{i+1, j}-U_{i, j}}{\Delta \xi} .
$$

For $j=2$ and $i=1$, value of function $U_{12}$ is determined from formula

$$
U_{12}=\frac{4 r U_{02}+6 r U_{22}+U_{11}}{1+10 r},
$$

where parameter $r=\Delta \tau / \Delta \xi=1 / p$. 
When $j>2$ then system of equations (13) can be written in the matrix form

$$
\mathbf{A} \cdot \mathbf{U}=\mathbf{B},
$$

where

$$
\begin{gathered}
\mathbf{A}=\left[\begin{array}{cccccc}
10 r+1 & -6 r & 0 & 0 & \ldots & 0 \\
-8 r & 18 r+1 & -10 r & 0 & \ldots & 0 \\
0 & -12 r & 26 r+1 & -14 r & \ldots & 0 \\
\ldots & \ldots & \ldots & \ldots & \ldots & \ldots \\
0 & 0 & \ldots & 0 & -4(j-1) r & (8(j-1)+2) r+1
\end{array}\right] \\
\mathbf{U}=\left[\begin{array}{c}
U_{1, j} \\
U_{2, j} \\
U_{3, j} \\
\cdots \\
U_{j-1, j}
\end{array}\right], \quad \mathbf{B}=\left[\begin{array}{c}
U_{1, j-1}+4 r U_{0} \\
U_{2, j-1} \\
U_{3, j-1} \\
\ldots \\
U_{j-1, j-1}+(4(j-1)+2) r U_{B}
\end{array}\right]
\end{gathered}
$$

\section{Numerical examples}

In this section, two examples of melting processes of ice and aluminum are considered. We apply the numerical scheme formulated above, perform calculations in curvilinear coordinates $(\xi, \tau)$ and then convert the results to standard Cartesian coordinates $(x, t)$.

Example 3.1. In the calculations we assume the following values of parameters for the melting process of ice: $u_{B}=273.15 \mathrm{~K}, u_{0}=298.15 \mathrm{~K}, l=0.1 \mathrm{~m}$, $c=4189.9 \mathrm{~J} /(\mathrm{kg} \mathrm{K}), \quad L=333400 \mathrm{~J} / \mathrm{kg}, \quad \rho=1000 \mathrm{~kg} / \mathrm{m}^{3}, \quad K=0.5664 \mathrm{~W} /(\mathrm{m} \mathrm{K})$. The calculation results are presented in graphs in Figures 1 and 2.

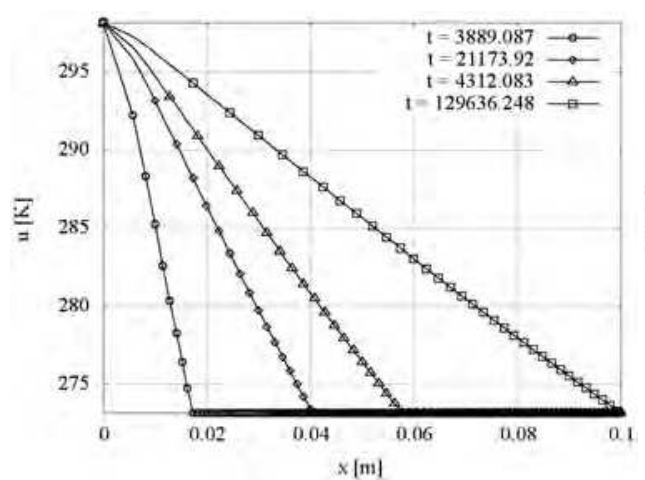

Fig. 1. The calculated temperature distribution in liquid region for Example 3.1 and $n=300$

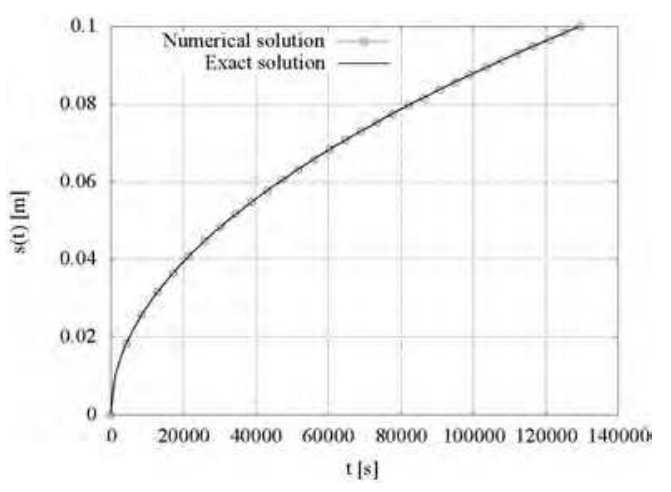

Fig. 2. The comparison of numerical results with the exact solution for Example 3.1 and $n=300$ 
Example 3.2. In the second example the melting process of aluminum is considered. The simulation assumes the following values of parameters: $u_{B}=931 \mathrm{~K}$, $u_{0}=1073 \mathrm{~K}, \quad l=0.1 \mathrm{~m}, \quad c=1130.44 \mathrm{~J} /(\mathrm{kg} \mathrm{K}), \quad L=396000 \mathrm{~J} / \mathrm{kg}$, $\rho=2380 \mathrm{~kg} / \mathrm{m}^{3}, \quad K=215 \mathrm{~W} /(\mathrm{mK})$. The calculation results are presented in graphs in Figures 3 and 4.

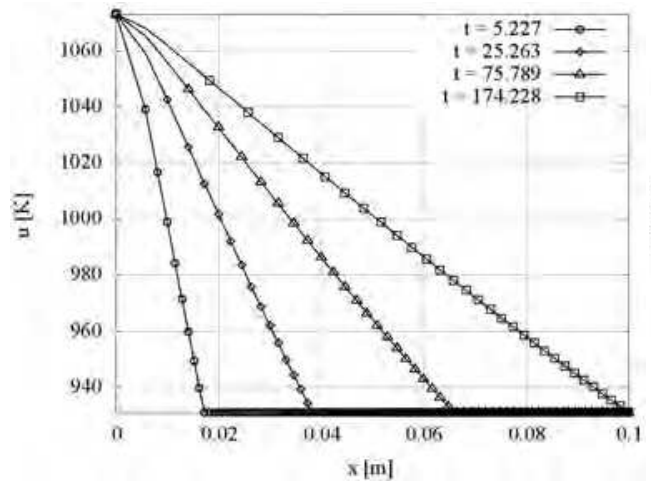

Fig. 3. The calculated temperature distribution in liquid region for Example 3.2and $n=300$

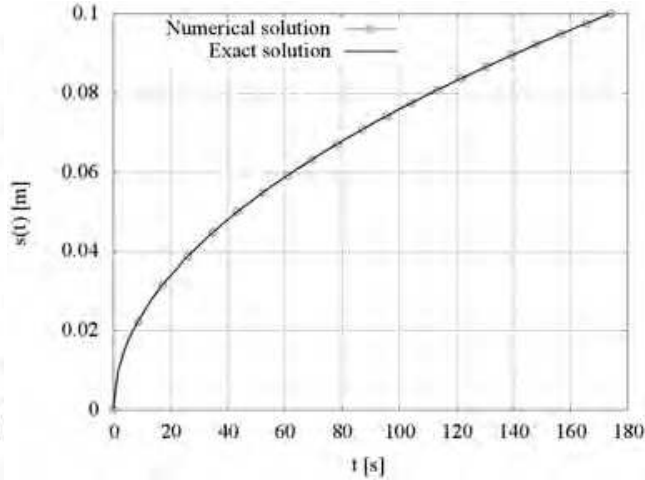

Fig. 4. The comparison of numerical results with the exact solution for Example 3.2 and $n=300$

\section{Conclusions}

In this paper we presented a new approach to solve the one-phase 1D Stefan problem. The introduced method is based on the finite difference method for the heat-flow equation in new space coordinates. This approach allows us to use a rectangular grid with the constant time and space steps. We compared numerical results obtained for 300 steps with the exact solution and received average relative error: $0.126 \%$ for Example 3.1 and $0.207 \%$ for Example 3.2 respectively.

Let us note that classical moving boundary problems were extensively studied in literature (compare [1-10] and the references given therein). Most of these problems can only be solved using numerical methods. The motivation of the proposed approach is its further application to fractional versions of the Stefan problem [11-14]. In the literature certain exact solutions to some simple cases of this problem were developed. However, in principle, they depend on parameters hidden in complicated function equations which are very difficult to solve. Similar to the standard theory of heat or solute transfer, the general fractional moving boundary problems could be solved only using numerical approach. However, the standard numerical schemes developed for classical Stefan problems are not effective in the case of models including fractional derivatives which are non-local operators. The constructed numerical scheme simplifies the grid and seems more suitable to model complicated non-local operators appearing in fractional moving 
boundary problems. The extension and application of the proposed method to the fractional case will be the subject of our further investigations.

\section{Acknowledgments}

This work was supported by the Czestochowa University of Technology Grant Number BS/MN 1-105-302/12/P.

\section{References}

[1] Stefan J., Ueber einige Probleme der Theorie der Waermeleitung, S.-B. Wien. Akad. Mat. Natur. 1889, 98,173-184.

[2] Crank J., Free and Moving Boundary Problems, Clarendon Press, Oxford 1984, 163-168.

[3] Gupta S.C., The Classical Stefan Problem. Basic Concepts, Modeling and Analysis, Elsevier, Amsterdam 2003.

[4] Schniewind J., Solution of the solidification problem of a one - dimensional medium by a new numerical method, The extent Journal of the Iron and Steel Institute 1963, July.

[5] Mochnacki B., Suchy J.S., Modelowanie i symulacja krzepnięcia odlewów, WN PWN, Warszawa 1993.

[6] Douglas J., Gallie T.M., On the numerical integration of a parabolic differential equation subject to a moving boundary condition, Duke Mathematical Journal 1955, 22, 557-571.

[7] Murray W.D., Landis F., Numerical and machine solutions of transient heat-conduction problems involving melting or freezing, J. Heat Transfer 1959, 81, 106-12.

[8] Crank J., The Mathematics of Diffusion, Clarendon Press, Oxford 1975.

[9] Landau H.G., Heat conduction in a melting solid, Quarterly of Applied Mathematics 1950, 8, 81-94.

[10] Wu Z., Luo J., Feng J., A novel algorithm for solving the classical Stefan problem, Thermal Science 2011, 15, 39-44.

[11] Liu Junyi, Xu Mingyu, An exact solution to the moving boundary problem with fractional anomalous diffusion in drug release devices, Z. Angew. Math. Mech. (ZAMM) 2004, 84, 22-28.

[12] Liu Junyi, Xu Mingyu, Some exact solutions to Stefan problems with fractional differential equations, J. Math. Anal. Appl. 2009, 351, 536-542.

[13] Chen Yin, Xicheng Li, Anomalous diffusion of drug release from slab matrix: Fractional diffusion models, Int. J. Pharmaceutics 2011, 418, 78-87.

[14] Voller V., An exact solution of a limit case Stefan problem governed by a fractional diffusion equation, Int. J. Heat Mass Transfer 2010, 53, 5622-5625. 